\title{
Segurança/insegurança alimentar em famílias urbanas e rurais no estado do Amazonas: I. Validação de metodologia e de instrumento de coleta de informação
}

\author{
Lúcia K. O. YUYAMA ${ }^{1}$, Jaime P.L. AGUIAR ${ }^{1}$, Lílian PANTOJA루, Roberto N. MAEDA ${ }^{1}$, Tatiana MELO ${ }^{1}$, \\ Fernando H. ALENCAR ${ }^{1}$, Angela M. Matos NASCIMENTO ${ }^{2}$, Neide M. Almeida NEGREIROS ${ }^{2}$, Ana \\ Maria Segall CORRÊA 3 , Rafael PÉREZ-ESCAMILLA ${ }^{4}$.
}

\section{RESUMO}

O presente estudo validou a metodologia e o instrumento de coleta de informação para análise da segurança/insegurança alimentar, em famílias urbanas e rurais no estado do Amazonas conforme o proposto pelo USDA (Departamento de Agricultura dos Estados Unidos). Valendo-se de amostra intencional de domicílios, selecionadas para representar estratos sociais diferentes foram computadas 194 famílias sendo 174 com crianças na área urbana de Manaus, envolvendo os seguintes bairros: Jesus me Deu, Novo Israel, Cidade Nova, Coroado e Conjunto Petro. Na área rural foram entrevistadas 209 famílias ribeirinhas e destas 131 com crianças, distribuídas entre os Municípios de Iranduba e Manacapuru. A validação final do questionário (Consistência interna global) deu-se por meio da comparação dos níveis de segurança e insegurança alimentar, com os estratos definidos dos indicadores sociais e de consumo. Podese concluir que os grupos com maior insegurança alimentar foram os situados em estratos sociais mais baixos e de baixo consumo de alimentos sensíveis a estas condiçôes. $\mathrm{O}$ instrumento de coleta apresentou alta validade e consistência interna.

\section{PALAVRAS-CHAVE}

Segurança alimentar, insegurança alimentar, validação quantitativa.

\section{Nutritional security/insecurity in urban and rural families of Amazonas state: Validation of methodology and of information collecting instrument}

\begin{abstract}
The present study validates the methodology and the information collecting instrument for analysis of nutritional security/insecurity on the urban and rural family level, proposed by the USDA (United States Department of Agriculture). An intentional sample of domiciles was selected to represent different social strata, 194 families were enrolled in urban Manaus, of which 174 had children, in the following neighborhoods: Jesus me Deu, Nova Israel, Cidade Nova, Coroado and Conj. Petro. In the rural area between the municipalities of Iranduba and Manacapuru, 209 riparian families were interviewed, and of these 131 had children. The final validation of the questionnaire (global internal consistency) was made by comparing, the levels of nutritional security/insecurity, with the defined social strata and food consumption indicators. The results demonstrated that the groups of highest nutritional insecurity were the very poor. The instrument presented high validation and internal consistency.
\end{abstract}

KEYWORDS

Nutritional security, nutritional insecurity, quantitative validation.

\footnotetext{
1 Instituto Nacional de Pesquisas da Amazônia, Coordenação de Pesquisas em Ciências da Saúde. Av. André Araújo, 2936, Aleixo, Manaus, AM. CEP: 69060-001. e-mail yuyama@inpa.gov.br

2 Secretaria de Estado de Saúde do Amazonas. Av. André Araújo 701, Aleixo, CEP. 69060-001, Manaus, AM.

${ }^{3}$ Universidade de Campinas, Departamento de Medicina Preventiva e Social. Faculdade de Ciências Médicas - Rua Tessália vieira de Camargo No 126, UNICAMP - Cidade Universitária "Zeferino Vaz". Barão Geraldo - Campinas - SP. CEP 13 084-970

${ }^{4}$ University of Connecticut, Department of Nutritional Sciences, Storrs, CT 06269-40
} 


\section{INTRODUÇÃo}

A inexistência de metodologia e instrumentos nacionais, até 2003, para análise da segurança/insegurança alimentar, acompanhamento e avaliação do impacto das políticas públicas pertinentes, particularmente o projeto "Fome Zero" fomentou a busca por ferramentas de fácil aplicação e que pudessem gerar informaçōes rápidas que subsidiariam o Governo nos seus esforços de formulação de políticas e ações de combate à fome.

A segurança alimentar é definida como acesso contínuo a quantidade e qualidade suficientes de alimentos, obtido por meio social aceitável, garantindo-se, desta forma, o bem estar e a saúde às pessoas. O Projeto "Fome Zero" (Instituto Cidadania, 2001) considera o direito à alimentação parte dos direitos civis básicos da população e um meio de se alcançar a cidadania plena. Propóe intervençôes variadas que visa abordar desde questōes estruturais como a melhoria de renda das famílias e o aumento da oferta de alimentos básicos, por meio do estímulo à agricultura familiar, até açōes específicas e diretas de assistência alimentar. Atualmente, suas açôes priorizam os municípios mais carentes do semi-árido nordestino.

A abrangência da política de combate à fome, a grandiosidade de seus objetivos, aliadas ao compromisso pessoal do Presidente do Brasil com sua implementação, exigem acompanhamento cuidadoso que possibilite, não apenas, a medida de seus efeitos sobre a segurança alimentar da população brasileira, mas também, o redirecionamento de suas ações.

Deve-se considerar que a preocupação com a segurança alimentar não é prerrogativa de países pobres, apesar de ser, em geral, uma emergência nestas naçôes. Nos Estados Unidos foi desenvolvido e aplicado um instrumento para análise de situação de insegurança alimentar (Frongilo,1999; Radimer, 2002; Cohen, 2005), tanto em populaçōes anglo-saxônicas quanto em populaçōes hispânicas, negras e asiáticas (Perez-Escamilla, 2003). Este mesmo instrumento foi adaptado para uso na Indonésia (Studdert et al., 2001) e zonas rurais do Senegal (PerezEscamilla, 2003).

O resultado da análise de segurança alimentar nos Estados Unidos ajudou a mudar a atitude dos legisladores e da população em geral que julgavam impossível haver insegurança alimentar em um país onde a obesidade é muito freqüente na população de baixo nível social e econômico (Studdert et al., 2001).

No Brasil há um grande esforço no sentido de validar o instrumento de coleta de informaçôes liderado por Segall-Corrêa et al. (2004). Nesse contexto, compondo o estudo de abrangência nacional, a presente proposta de investigação objetivou validar o instrumento de medição da insegurança alimentar, intra-familiar, na área urbana de Manaus e na área rural dos Municípios de Iranduba e Manacapuru, Calha do Rio Solimões.

\section{MATERIAL E MÉTODOS}

O presente estudo compôs um projeto de abrangência nacional de validação de metodologia e de instrumento de coleta de informação para análise da Segurança/Insegurança Alimentar, em nível familiar sob a Coordenação da UNICAMP e Universidade de Cornell com a participação de quatro Instituições de Ensino e Pesquisa: Universidade Estadual de Campinas, Universidade de Brasília, Universidade Federal da Paraíba e Instituto Nacional de Pesquisa da Amazônia.

Para a realização dos procedimentos de adequação trabalhouse com metodologias qualitativa e quantitativa, por meio de painéis multidisciplinares de especialistas e de grupo focais com representantes da comunidade da área urbana e rural, conforme o proposto por investigação realizada na Universidade de Cornell e amplamente utilizada pelo Departamento de Agricultura dos Estados Unidos (RADIMER, 2002; USDA, 2003). Foram viabilizados dois painéis na região amazônica, com participação de profissionais de diversas áreas do conhecimento. O conteúdo explorado nas dinâmicas com os grupos focais foi o proposto nos painéis dos especialistas, do mesmo modo que as versōes do questionário.

O instrumento testado incluiu 15 perguntas validadas em populações urbanas e rurais para analisar a segurança alimentar (SA) em três níveis: a) SA na família; b) SA dos adultos na família; c) SA das crianças e esta foi dividida em dois níveis de intensidade - "insegurança alimentar" e "fome".

No inquérito Urbano e Rural, após a aprovação pelo Comitê de Ética da Unicamp, por tratar-se de um estudo multicêntrico, optou-se por amostras intencionais de domicílios, selecionadas para representar quatro estratos de famílias com níveis sociais diferentes, segundo sua relação com a renda. Para tanto, 194 pessoas responsáveis pelas famílias, foram entrevistadas, sendo a amostra constituída pelas 174 famílias com crianças, envolvendo os seguintes bairros: Jesus Me Deu, Novo Israel, Cidade Nova, Coroado e Conjunto Petro.

$\mathrm{Na}$ área rural foram entrevistados 209 ribeirinhos e destes compuseram a amostra os 131, chefes de famílias com crianças, nos Municípios de Iranduba e Manacapuru- Calha do Rio Solimões. A partir de escores de respostas positivas, as 15 perguntas do questionário estabeleceram-se os gradientes de IA com os seguintes pontos do corte: segurança alimentar: escore 0 ; IA leve: escore de 1 a 5; IA moderada: escore de 6-10; IA severa: escore de 11 a 15 .

A consistência interna dos itens da escala de insegurança alimentar foi testada por meio do coeficiente alfa de Chronbach, considerando-se como adequados, neste trabalho, valores superiores a 0,85 . A validação final do questionário (consistência interna global) deu-se por meio da comparação dos níveis de segurança e insegurança alimentar com os estratos definidos dos 
indicadores sociais e de consumo (Albert \& Sanjur, 2000; PerezEscamilla, 2003).

\section{RESULTADOS E DISCUSSÃO}

$\mathrm{Na}$ área urbana de Manaus as maiores concentrações de famílias entrevistadas situaram-se nos bairros de Novo Israel e Jesus me Deu ( $29 \%$ cada). Na zona rural o maior percentual de famílias entrevistadas esteve centrado em Iranduba mais especificamente na Comunidade de Jandira (49\%) seguida do Lago do Limão (21\%).

Quanto ao perfil demográfico das famílias chama a atenção o fato da ausência de crianças em $47 \%$ das famílias entrevistadas, no meio rural, indicando possivelmente a opção de adultos e aposentados viver no interior.

Entre as famílias incluídas na amostra, a análise de renda em relação ao salário mínimo (SM) mostrou que a seleção intencional da amostra foi adequada, pois as diferentes classes de renda estavam presentes (Figuras $1 \mathrm{e} 2$ ). Contudo, o maior percentual em função da distribuição de renda concentrou-se entre $>1 \mathrm{SM}$ $<3 \mathrm{SM}$, sobretudo na área rural. No questionário aplicado à população urbana e rural incluíram-se, como recursos para os procedimentos de validação externa, indicadores de renda e de consumo diário de alimentos. O questionário apresentou validação preditiva adequada, tanto na área urbana quanto na rural. Isto pode ser observado pelo paralelismo das quatro curvas relativas aos estratos de renda e pelas diferenças de prevalência de respostas positivas, segundo o gradiente de insegurança alimentar que estas perguntas traduzem (Figuras $3 \mathrm{e} 4$ ).

Na Tabela 1 observa-se a prevalência de IA nas duas amostras estudadas. Os gradientes de insegurança alimentar mostram, para o total de famílias, da área urbana e rural, comportamento consistente de tendência de menor prevalência de insegurança alimentar na zona urbana de Manaus, demonstrado tanto pela menor freqüência de IA Grave como maior segurança (AS) na área rural. Contudo, há necessidade de estudos com amostras representativas nas áreas estudadas para confirmar tais tendências.

Nas áreas estudadas foram observadas relações inversas entre IA em função dos estratos de renda. Esta relação é expressiva nas

Tabela 1 - Classificação da segurança alimentar (SA) e insegurança alimentar (IA) em área urbana de Manaus e rural nos Municípios de Iranduba e Manacapuru.

\begin{tabular}{ccc}
\hline Gradiente de IA & Área urbana (\%) & Área rural (\%) \\
\hline SA (escore $=0)$ & 10,9 & 31,3 \\
IA Leve (escore $=1-5)$ & 24,6 & 22,9 \\
IA Moderada (escore $=6-10)$ & 20,0 & 17,6 \\
IA Severa (escore $=11-15)$ & 44,6 & 28,2 \\
Número de famílias com & 174 & 131 \\
\hline crianças &
\end{tabular}

$\mathrm{SA}=$ Segurança Alimentar; $\mathrm{IA}=$ Insegurança Alimentar

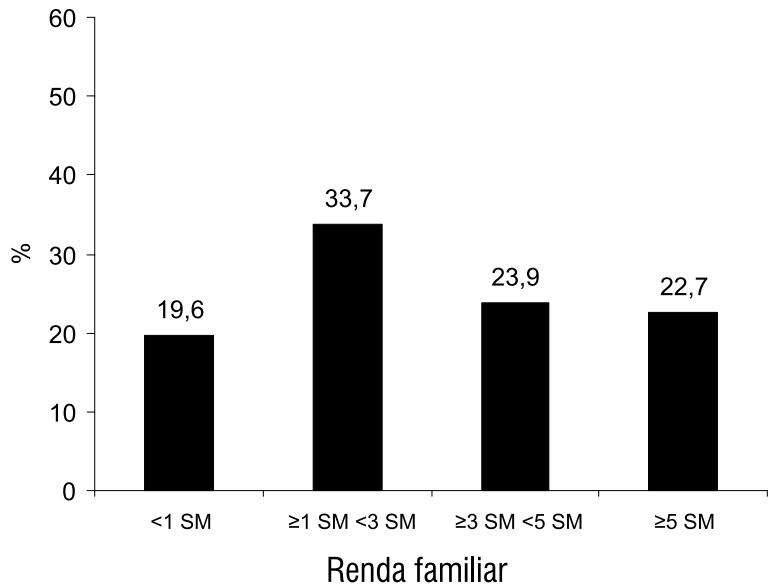

Figura 1 - Distribuição das famílias avaliadas quanto à segurança/insegurança alimentar de segundo a renda (total) de famílias da área urbana de Manaus, AM. SM = salário mínimo.

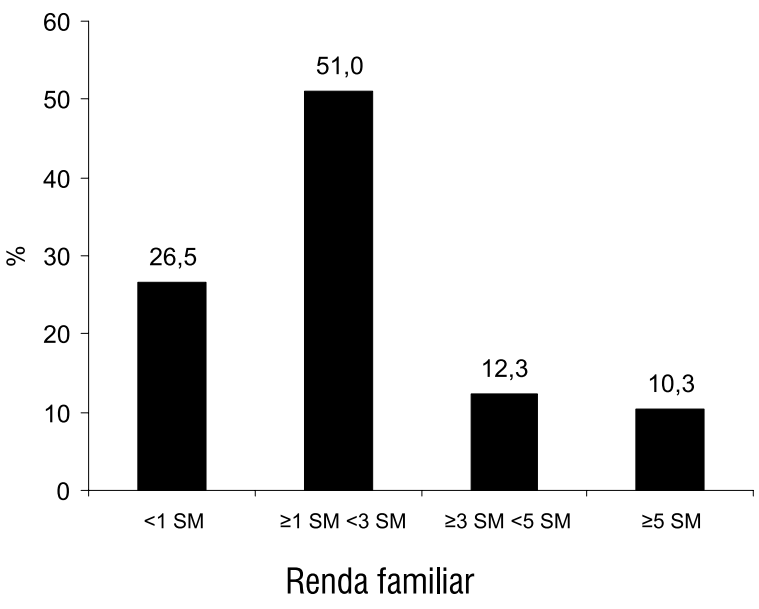

Figura 2 - Distribuição das famílias avaliadas quanto à segurança/insegurança alimentar segundo a renda (total) nas áreas rurais dos Municípios de Iranduba e Manacapuru, AM. SM - Salário mínimo.

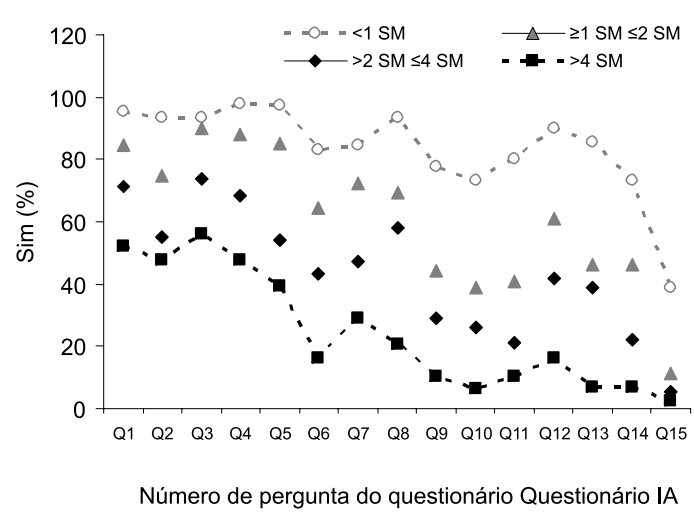

Figura 3 - Insegurança alimentar na área urbana de Manaus, AM, segundo a renda total. $(\mathrm{n}-174)$. $\mathrm{SM}=$ salário mínimo. 


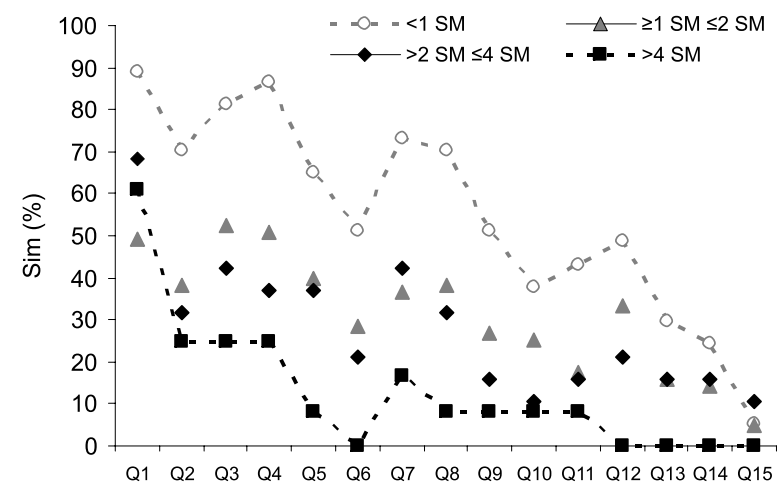

Número de pergunta do questionário Questionário IA

Figura 4 - Insegurança alimentar na área rural dos Municípios de Iranduba e Manacapuru, AM, segundo a renda total. (n-131). SM = salário mínimo.

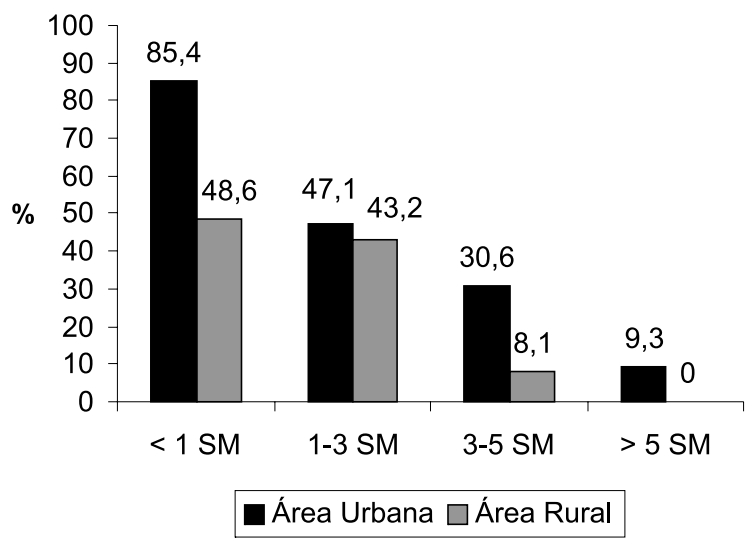

Figura 5 - Insegurança alimentar em diferentes estratos de renda na área urbana de Manaus ( $n=174)$ e rural nos Municípios de Iranduba e Manacapuru ( $\mathrm{n}=131)$. SM = salário mínimo.

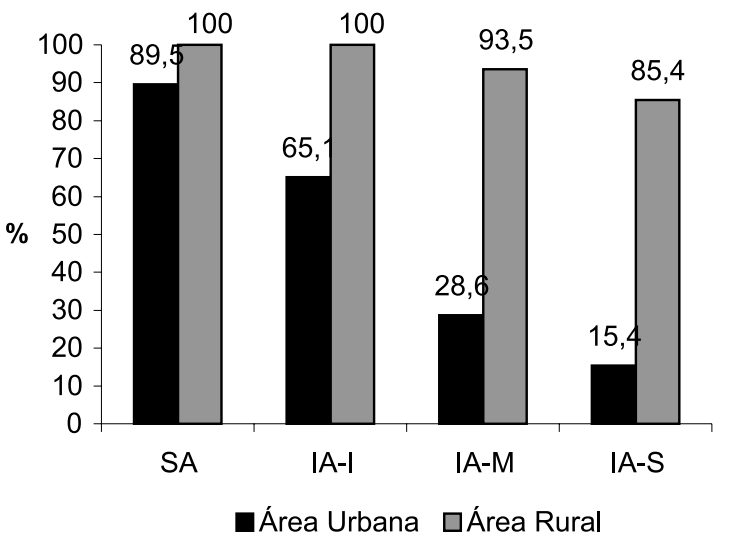

Figura 6 - Consumo diário de frutas por famílias da área urbana de Manaus $(n=174)$ e das áreas rurais dos Municípios de Iranduba e Manacapuru $(n=131)$, segundo 0 nível de Insegurança Alimentar. SA - segurança alimentar, IA-I - insegurança alimentar leve, IA-M - insegurança alimentar moderada e IA-G insegurança alimentar grave. áreas urbana e rural, particularmente na área urbana. Ao menor nível de renda corresponde o maior nível de insegurança alimentar (Figura 5). Constatou-se prevalência de IA/Grave no estrato inferior de rendimento familiar (menos de $1 \mathrm{SM}$ ), variando entre 85,4\% e 48,6\% na área urbana e rural respectivamente, conforme Figuras (5). Não obstante, Pérez-Escamilla et al. (2004) apresentaram resultados similares quando da avaliação do módulo da segurança e insegurança alimentar em Campinas. $\mathrm{O}$ comportamento consistente e válido do referido módulo reproduziu-se em dois inquéritos independentes, um em Campinas, com amostra de 847 famílias, representativas dos domicílios urbanos da cidade (Panigassi, 2005) e outro em Brasília com amostra de 1000 famílias com crianças menores de seis anos (Leão, 2005). As tendências confirmam não só a consistência geral do questionário e a pertinência de seu uso como medida direta de IA em famílias, mas também a relevância destes resultados do ponto de vista social e político e a necessidade de medidas de intervenção.

Os gradientes de IA também estiveram associados com padrōes diferentes de consumo diário de alimentos pela pessoa entrevistada. Famílias classificadas como experimentando IA Grave, apresentam-se com menor probabilidade de consumo diário de leite e derivados de leite, área urbana e rural, frutas e verduras, área urbana, ovos área urbana e rural e feijão e revelam que estes alimentos são nestas populações sensíveis à insegurança alimentar (Figuras 6 e 7). Portanto, os alimentos sensíveis a insegurança alimentar na área rural foram: leite, derivados do leite e não sensíveis: cereais, frutos, verduras, raízes, óleo e carne. Tais constataçôes reforçam a hipótese de que é no interior que os ribeirinhos sobrevivem com mais dignidade, pela oportunidade, do plantio de verduras e fruteiras de fundo de quintal dentre elas: mamão, abacaxi, ingá (Inga capitada) e manga. Soma-se ainda a disponibilidade de outras fruteiras nativas como tucumã (Astrocaryum aculeatum), buriti (Mauritia flexuosa), açaí (Euterpe

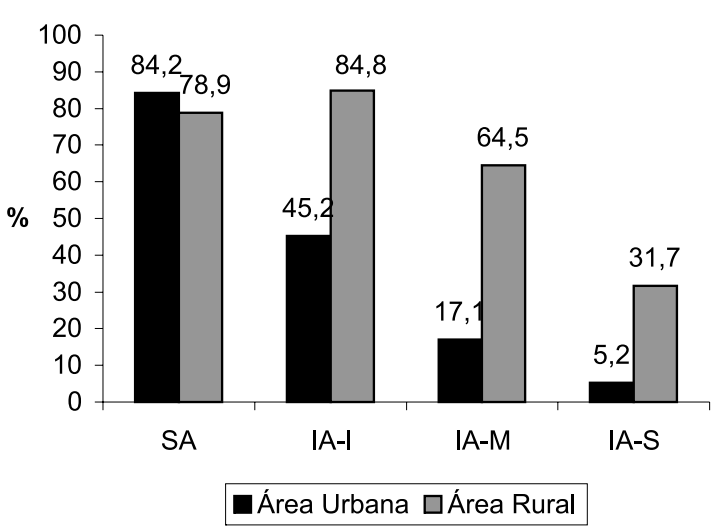

Figura 7 - Consumo diário de derivados do leite segundo o nível de Insegurança Alimentar. SA - segurança alimentar, IA-I - insegurança alimentar leve, IA-M - insegurança alimentar moderada e IA-G insegurança alimentar grave. 


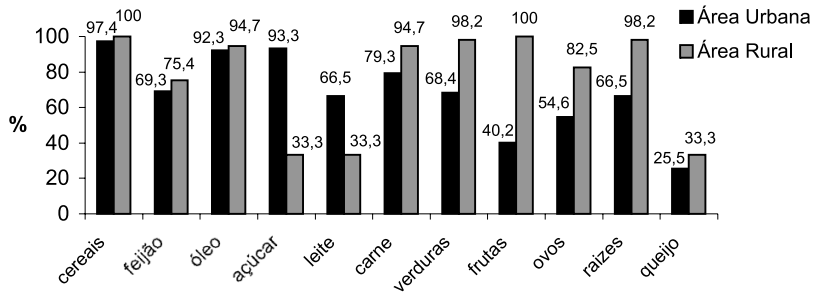

Figura 8 - Alimentos consumidos pelo menos uma vez ao dia por famílias da área urbana de Manaus e das áreas rurais dos Municípios de Iranduba e Manacapuru, AM.

oleracea Mart.) e pupunha (Bactris gasipaes Kunth), o que permite, dependendo da sazonalidade, maior oferta e consumo, assim como a oportunidade da pesca e caça. Essa diversidade de alimentos é que faz a diferença quanto à sensibilidade comparada à área urbana. $\mathrm{O}$ fato do consumo de frutas, na zona rural, não mostrar relação com IA grave, sugere maior reflexão sobre a aplicação deste parâmetro em situações rurais com abundante oferta de frutas catados e/ou coletados.

$\mathrm{Na}$ Figura 8 são mostrados os alimentos consumidos pelo menos uma vez ao dia pelas famílias das áreas urbana e rural, o que demonstra a situação mais privilegiada dos ribeirinhos de cidades do interior em relação ao consumo de frutas, ovos e raízes, contrapondo com o baixo consumo de açúcar e leite, quando relacionado com a área urbana de Manaus.

Estas observaçôes reafirmam o poder preditivo do questionário, bem como a consistência das perguntas que originam os escores. A consistência interna da escala Alpha de Chronbach (validade psicométrica), demonstrou em todas as amostras valores superiores a 0,85, ou seja, um Alpha de Chronbach de 0,946 na área urbana e área rural 0,952.

\section{CONCLUSÃO}

Os grupos de maior insegurança alimentar são os situados em estratos sociais de maior pobreza e de baixo consumo de alimentos sensíveis a estas condiçôes. Portanto, este procedimento permitiu observar o caráter preditivo do questionário demonstrando alta validade do questionário em Manaus. Foi constatada validade do conteúdo (painel de especialista), de conceitos e compreensão (Grupo focal da comunidade), validade preditiva e alta consistência interna.

\section{AGRADECIMENTOS}

Ao Ministério da Saúde, Organização Pan-Americana da Saúde (OPS-Brasil), Universidade Estadual de Campinas, Instituto Nacional de Pesquisas da Amazônia, Secretaria de Estado de Saúde do Amazonas, AM pelo apoio Financeiro e logístico. As
Universidades Nilton Lins e UNIP pela colaboração e apoio. Aos técnicos e alunos que colaboraram na aplicação dos questionários. Em especial a Dra. Helyde A. Marinho quando da participação na Oficina com o grupo de especialistas.

\section{BIBLIOGRAFIA CITADA}

Albert, P.L; Sanjur, D. 2000. The adaptation and validation of the food security scale in a community of Caracas, Venezuela. Arch. Latinoam. Nutr., 50(4):334-340.

Cohen, B. 2005. Community Food Security Assessment Toolkit. ERS E-FAN No. 02-013. 2002. p. 166. (http:// www.ers.usda.gov/publications/efan02013/). Acesso: 23/04/ 2005.

Frongillo, E.A. 1999. Validation of measures of food insecurity and hunger. J Nutr., 129 (Suppl. 2): 506-509.

Instituto Cidadania. 2001. Projeto Fome Zero: uma politica de segurança alimentar para o Brasil. São Paulo: Instituto da Cidadania. 118pp.

Leão, M. 2005. Segurança Alimentar e Risco de Sobrepeso e Obesidade em famílias de crianças menores de 6 anos. Dissertação Mestrado. Universidade de Brasília, Brasília, DF. 121pp.

Panigassi, G. 2005. Inquérito populacional sobre a percepção da segurança alimentar intrafamiliar no município de Campinas, SP. Tese de Doutorado, Universidade Estadual de Campinas, Campinas, São Paulo. 188pp.

Perez-Escamilla, R.; Randolph, S.; Hathie, I.; Gaye, I. 2004. Adaptation and validation of the USDA food security scale in rural Senegal. FASEB J., 18:A106.

Perez-Escamilla, R.; Segall-Corrêa, A.M.; Maranha, L.K.; Sampaio, M.F.A; Marin-León, L.; Panigassi, G. 2004. An adapted version of the U.S. Department of Agriculture Food Insecurity Module is a valid tool for assessing household food insecurity in Campinas, Brasil. J. Nutr., 134:1923-1928.

Radimer, K.L. 2002. Measurement of household food security in the USA and other industrialised countries. Public Health Nutr., 5(6A):859-864.

Segal-Corrêa, A.M.; Escamilla, R.P.; Maranha, L.K.; Sampaio, M.F.A. 2004. Relatório Técnico. Versão preliminar Acompanhamento e avaliação da Segurança alimentar de famílias brasieleiras:Validação de Metodologia e de instrumento de coleta de informação. Urbano/ rural. UNICAMP. Campinas. 33pp.

Studdert; L.J.; Frongillo, E.A.; Valois, P. 2001. Household food insecurity was prevalent in Java during Indonesia's economic crisis. J Nutr., 131(10): 2685-2691.

United Stater Department of Agriculture. 2003. Food security in the United States: history of the food security measurement project. (http://www. ers.usda.gov/briefing/foodsecurity/ history). Acesso: 22/08/2003.

Recebido em 29/09/2005

Aceito em 28/02/2007 
